\title{
Laser Control of Self-Organization Process in Microscopic Region and Fabrication of Fine Microporous Structure
}

\author{
Yukimasa Matsumura, ${ }^{1}$ Wataru Inami, ${ }^{2,3}$ and Yoshimasa Kawata ${ }^{1,3}$ \\ ${ }^{1}$ Graduate School of Science and Technology, Shizuoka University, Hamamatsu, Shizuoka 432-8561, Japan \\ ${ }^{2}$ Division of Global Research Leaders, Shizuoka University, Hamamatsu, Shizuoka 432-8561, Japan \\ ${ }^{3} J a p a n$ Science and Technology Agency, CREST, Shiyod-ku, Tokyo 103-0075, Japan \\ Correspondence should be addressed to Yukimasa Matsumura, f5945008@ipc.shizuoka.ac.jp \\ Received 14 February 2011; Accepted 18 October 2011 \\ Academic Editor: Dieter Schuöcker
}

Copyright (c) 2012 Yukimasa Matsumura et al. This is an open access article distributed under the Creative Commons Attribution License, which permits unrestricted use, distribution, and reproduction in any medium, provided the original work is properly cited.

We present a controlling technique of microporous structure by laser irradiation during self-organization process. Selforganization process is fabrication method of microstructure. Polymer solution was dropped on the substrate at high humid condition. Water in air appears dropping air temperature below the dew point. The honeycomb structure with regularly aligned pores on the film was fabricated by attaching water droplets onto the solution surface. We demonstrate that it was possible to prevent forming pores at the region of laser irradiation and flat surface was fabricated. We also demonstrated that a combination structure with two pore sizes and flat surface was produced by a single laser-pulse irradiation. Our method is a unique microfabrication processing technique that combines the advantages of bottom-up and top-down techniques. This method is a promising technique that can be applied to produce for photonic crystals, biological cell culturing, surface science and electronics fields, and so forth.

\section{Introduction}

Microfabrication techniques have been studied by many researchers with the developments of nanotechnologies [1]. Microfabrication techniques are categorized as topdown or bottom-up fabrication techniques. The top-down fabrication technique creates nanoscale and submicron scale devices by using externally controlled equipment to direct their assembly. One of typical top-down methods is photolithography. The advantages of the top-down methods are high precision and high reproducibility in the fabrication process [2]. The top-down method, however, requires expensive equipment, long processing time, and complicated procedures.

On the other hand, the bottom-up fabrication technique builds smaller components to more complex assemblies. The bottom-up techniques are known as self-organization process. The bottom-up methods have the advantages of short process time and low cost. They easily produce a uniform pattern in large area, while the arbitrary structures are difficult to be produced by bottom-up techniques.

It has been reported that microporous films are formed by the cast on the glass substrate under high humid condition by self-organization process of solution casting method [317]. The microstructures by self-organization process are expected to be used in many applications as follows. The microporous structure of regular aligned pores is made of polystyrene, and the line defect in this microporous structure is applied for photonic crystal [18, 19]. Micropatterned substrates by self-organization process are used as potential scaffolds for regeneration of cardiac and fibrous tissues [2025]. Pincushion films are prepared by peeling off the top layer of the self-organization honeycomb film, and metalized pincushion films are fabricated by electroless plating. These metalized pincushion structures are expected to application of field emitter arrays [26].

We propose controlling self-organization process by laser irradiation in order to produce arbitrary structures. It is a 


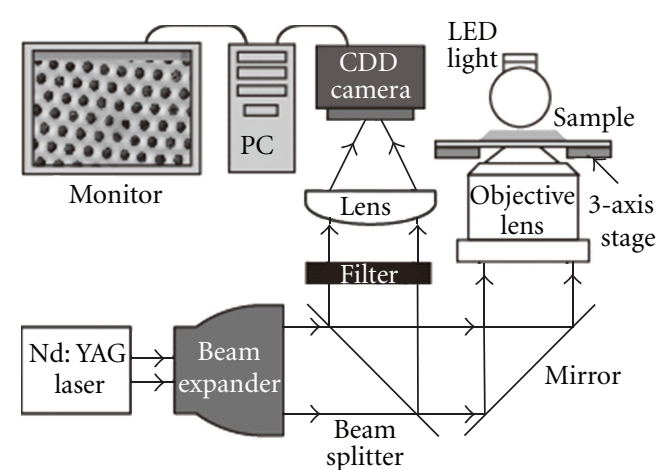

FIGURE 1: Optical setup for controlling of self-organization process.

unique technique that combines top-down and bottom-up techniques.

\section{Laser Control of Self-Organized Process}

2.1. Fabrication of Self-Organized Microporous Films. It has been reported that honeycomb microporous films are fabricated by self-organization process [3-17]. The mechanism of fabrication process of honeycomb structure on a film is explained as follows.

Polymers with hydrophilic group are dissolved in highly volatile solvent. This solution is dropped on a glass substrate in high humid air. Air near the solution surface is cooled because the solvent draw heat during evaporation. Because the air temperature drops below the dew point, the water droplets are generated near the solution surface and they attach on the surface. Water droplets on the solution surface are packed as honeycomb structure by capillary force. Finally, the solvent and the water droplets are completely evaporated and microporous droplets are produced at the position that the water droplets are attached on the solution surface.

We used a solution of polystyrene which was monocarboxy-terminated (weight-average molecular weight: 50,000, Scientific Polymer Products, Inc.) in chloroform (Kanto chemical co., Inc.). This polymer has carboxyl group as hydrophilic group. The polystyrene chloroform solution of $25-50 \mu \mathrm{L}$ was dropped onto the cover glass $(24 \times 40 \mathrm{~mm}$, Matsunami glass Ind., Ltd.) at high humid condition of relative humidity $75 \%$. Herewith, the polystyrene layer of about $15 \mu \mathrm{m}$ thickness with microporous structure was fabricated. The fabricated microporous films were observed with scanning electron microscope (SEM; JSM-6390, JEOL Ltd.) after sputtering of gold of $20 \mathrm{~nm}$ thickness.

2.2. Laser Control of Self-Organization Process. Figure 1 shows the optical setup to control self-organization process by laser irradiation during fabrication of microporous film. A Q-switched Nd: YAG laser is used as a light source. Wavelength, pulse width, and repetition frequency of the laser are $532 \mathrm{~nm}, 3-5 \mathrm{~ns}$, and $1 \mathrm{~Hz}$, respectively. Laser light is focused into the solution on the cover glass by an objective lens. The solution thickness decreases because of solvent evaporation, and the solution surface is moved in the optical

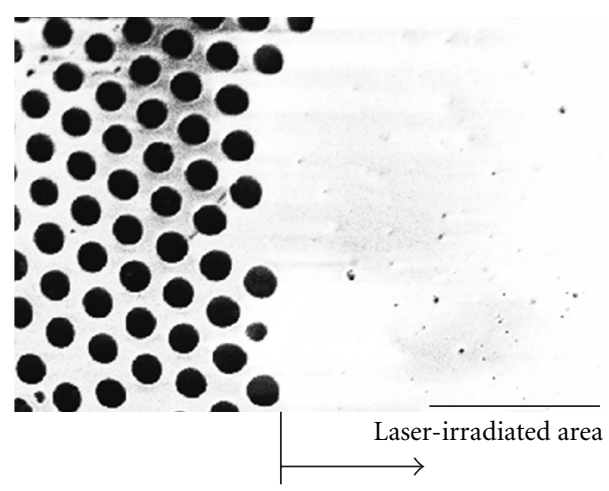

FIGURE 2: SEM image of controlling self-organized microporous polystyrene film by laser irradiation from below. Scale bar: $20 \mu \mathrm{m}$.

axis direction during self-organization process. Therefore we tracked the solution surface with moving the glass substrate in the optical axis direction by the 3 -axis stage. The solution droplet surface is observed by a CCD camera with the illumination of an LED light.

\section{Experimental Demonstration of Laser Control of Self-Organization Process}

3.1. One Pulse Laser Irradiation from Bottom Side of the Solution. The surface of solution was laser-irradiated from bottom side after 53 seconds from the solution being dropped on the cover glass. The solution concentration is $40 \mathrm{~g} / \mathrm{L}$ and drip amount is $25 \mu \mathrm{L}$. Numerical aperture of objective lens is 0.4. Laser pulse energy is $15 \mu \mathrm{J}$. Figure 2 shows SEM images of self-organization microporous polystyrene film with irradiation of a laser pulse. The figure shows that the plane surface without pores was fabricated by the laser irradiation in the microporous structure during selforganization process. If laser light is irradiated after finishing self-organization process, the self-organization microporous films are damaged by the laser pulse.

We can recognize clearly that plane surface without pores area was produced by the laser irradiation. The result shows that we can prevent forming pores at the laser irradiated area. In fact, we may say that self-organization process can be controlled by a pattern of the laser irradiation and planar surface of arbitrary pattern such as line is fabricated in the microporous structure.

We discuss the mechanism that the water droplets on the solution surface are removed by the laser irradiation. Figure 3 shows the illustration of mechanism of laser controlling of self-organization process. We believe that the shock wave is main factor to remove the water droplets. When the laser light is irradiated to a solution and the irradiation intensity exceeds the threshold value, the shock wave is induced with the laser ablation pressure [27]. The shock wave exceeds the solution surface tension, and the water droplets are removed at the laser-irradiated region. Because the solution is irradiated by a laser pulse just before it is completely evaporated, there no enough time water droplets are attached 


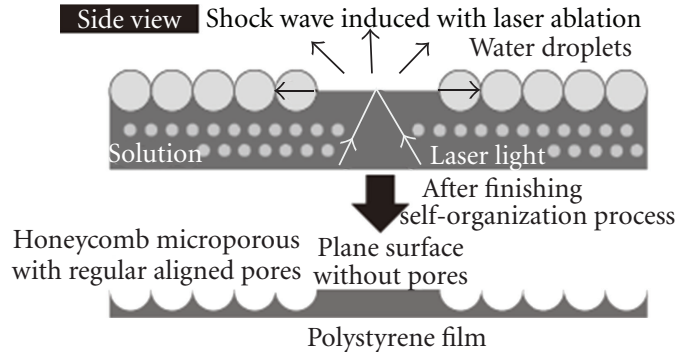

FIGURE 3: Side view pattern diagram of solution surface with laser irradiation.

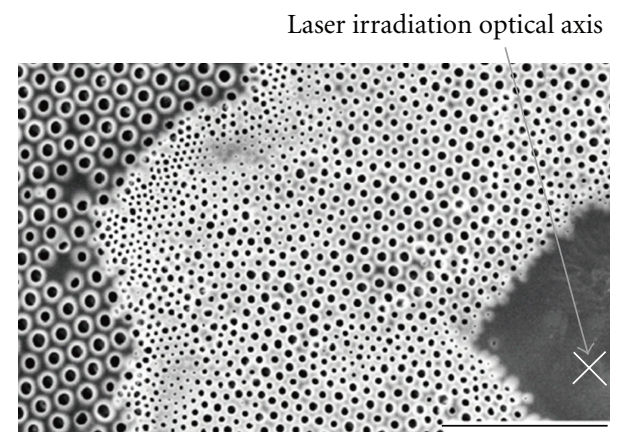

Figure 4: SEM image of two pore sizes and plane surface polystyrene film by laser irradiation from below focusing inside solution. Scale bar: $50 \mu \mathrm{m}$.

again in this area and the plane surface without pores is generated.

\subsection{Fabrication of Microporous Structure by Focusing Laser} Light inside Solution Droplet. We found that it was possible to produce a combination of large and small pore sizes and plane surface without pores by focusing laser light inside the droplet solution. The solution thickness is about $50 \mu \mathrm{m}$ when the laser light is irradiated. The laser light is focused at the point of below about $15 \mu \mathrm{m}$ from the solution surface after 110 seconds from solution being dropped on the cover glass. The solution concentration is $35 \mathrm{~g} / \mathrm{L}$ and drip amount is $25 \mu \mathrm{L}$. Laser pulse energy is $300 \mu \mathrm{J}$.

Figure 4 shows SEM image of the combination structure produced by focusing laser light inside of solution and the optical axis is also shown in this figure. In the area near the optical axis plane surface without pores was produced. The small pores were fabricated at the region surrounding the plane surface area and regularly aligned pores with honeycomb structure also surround the area of small pores.

Figure 5 shows the illustration of mechanism of fabricating small pores and plane surface by the laser irradiation. The reason that the small water droplets were produced is described as follows. On self-organization process, polystyrene chloroform solution is evaporated and temperature at the solution surface is decreased by solvent evaporation. Because of temperature decrease at the solution surface, the convection occurrs in the solution by temperature difference between surface and inside of the solution.

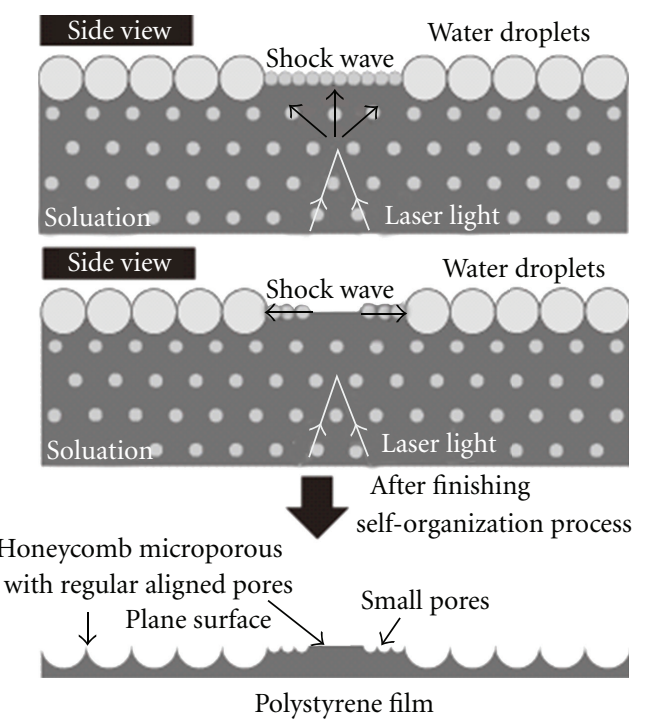

FIGURE 5: Side view pattern diagram of inside solution with laser irradiation.

Some water droplets on the solution surface are entered inside the solution by convection flow. The water droplets on the solution surface are grown due to constant moisture supplying, but the water droplets in the solution are not grown because no moisture was supplied to the droplets and small water droplets are kept in small size.

When the laser was focused inside the solution, the small water droplets inside the solution were pushed to the surface by the shock wave of the laser pulse irradiation. All water droplets were removed near the optical axis because the shock wave was strong enough. Because these two phenomena occurred at about the same time, the central area of the laser irradiation was the plane surface without pores and the surrounding area is small pores structure. Unaffected area by the laser irradiation is large pores structure.

3.3. One Pulse Laser Irradiation from the Top Side of the Solution. The surface of solution is irradiated by the laser from the top side after 90 seconds from the solution being dropped on the cover glass. Solution concentration is $40 \mathrm{~g} / \mathrm{L}$ and drip amount is $50 \mu \mathrm{L}$. Numerical aperture of objective lens is 0.42 . Laser pulse energy is $15 \mu \mathrm{J}$. Figure 6 shows SEM images of self-organization microporous polystyrene film with irradiation of a laser pulse. This picture shows that the plane surface without pores was fabricated in the microporous structure. Compared to the result of Figure 2, the plane surface area of Figure 6 is narrower. The plane surface area is reduced to about one-sixth. We may say that the irradiation of laser from the top side reduces the lasercontrolled area.

The reduction of controlled area by the laser irradiation from the top side was attributed to spherical aberrations. In the case of the laser irradiation from the bottom side, the laser light transmits the polystyrene chloroform solution, so the laser spot is aberrated during the propagation in the solution [28]. However, in the case of the laser irradiation 


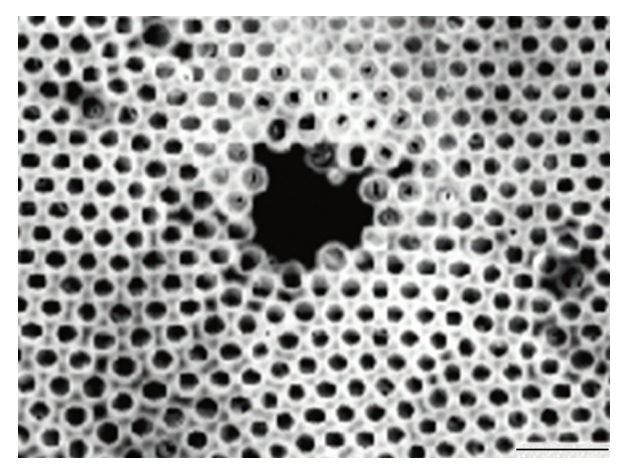

FIGURE 6: SEM image of controlling self-organized microporous polystyrene film by laser irradiation from above. Scale bar: $20 \mu \mathrm{m}$.

from the top side, there are no aberrations and the laser spot is well-focused on the solution surface.

\section{Conclusion}

We demonstrated that the microporous structure was able to be controlled by the laser light irradiation during selforganization process. The plane surface area without pores was produced by removing the attached water droplets from the surface with a shock wave induced with a laser pulse. When the laser light was irradiated the polystyrene chloroform solution from the top side of the solution, the area where the water droplets were removed was reduced. We also demonstrated that in the case of the laser irradiation from the bottom side of the solution, the combination structure of two pore size and plane surface was also fabricated by focusing inside the solution. In this case, the small water droplets inside the solution were pushed to the surface with the shock wave and the small water droplets on the solution surface were removed. Our method has a potential that combines the processes of bottom-up and top-down methods. It is a promising technique that can be applied to produce photonic crystals, biological culturing process, water repellent materials, electronic, and so on.

\section{References}

[1] M. Madou, Fundamental of Microfabrication, CRC Press LLC, 1997.

[2] T. Kawai, Graphic Explanation All of Application of Nanotechnology, Kogyochosakai, 2002.

[3] G. Widawski, M. Rawiso, and B. Francois, "Self-organized honeycomb morphology of star-polymer polystyrene films," Nature, vol. 369, no. 6479, pp. 387-389, 1994.

[4] N. Maruyama, T. Koito, J. Nishida et al., "Mesoscopic patterns of molecular aggregates on solid substrates," Thin Solid Films, vol. 327-329, no. 1-2, pp. 854-856, 1998.

[5] B. de Boer, U. Stalmach, H. Nijland, and G. Hadziioannou, "Microporous honeycomb-structured films of semiconducting block copolymers and their use as patterned templates," Advanced Materials, vol. 12, no. 21, pp. 1581-1583, 2000.

[6] M. H. Stenzel-Rosenbaum, T. P. Davis, and A. G. Fane, "Porous polymer films and honeycomb structures made by the self-organization of well-defined macromolecular structures created by living radical polymerization techniques," Angewandte Chemie International Edition, vol. 40, no. 18, pp. 34283432, 2001.

[7] M. Srinivasarao, D. Collings, A. Philips, and S. Patel, "Threedimensionally ordered array of air bubbles in a polymer film," Science, vol. 292, no. 5514, pp. 79-83, 2001.

[8] L. Song, R. K. Bly, J. N. Wilson et al., "Facile microstructuring of organic semiconducting polymers by the breath figure method: hexagonally ordered bubble arrays in rigid-rod polymers," Advanced Materials, vol. 16, no. 2, pp. 115-118, 2004.

[9] L. V. Govor, I. A. Bashmakov, F. N. Kaputski, M. Pientka, and J. Parisi, "Self-organized formation of low-dimensional network structures starting from a nitrocellulose solution," Macromolecular Chemistry and Physics, vol. 201, no. 18, pp. 2721-2728, 2000.

[10] Y. Tian, S. Liu, H. Ding, L. Wang, B. Liu, and Y. Shi, "Formation of deformed honeycomb-patterned films from fluorinated polyimide," Polymer, vol. 48, no. 8, pp. 2338-2344, 2007.

[11] Y. Xu, B. Zhu, and Y. Xu, "A study on formation of regular honeycomb pattern in polysulfone film," Polymer, vol. 46, no. 3, pp. 713-717, 2005.

[12] O. Pitois and B. François, "Crystallization of condensation droplets on a liquid surface," Colloid and Polymer Science, vol. 277, no. 6, pp. 574-578, 1999.

[13] H. Yabu, M. Kojima, M. Tsubouchi, S. Y. Onoue, M. Sugitani, and M. Shimomura, "Fabrication of photo-cross linked honeycomb-patterned films," Colloids and Surfaces A, vol. 284285, pp. 254-256, 2006.

[14] X. Zhao, Q. Cai, G. Shi, Y. Shi, and G. Chen, "Formation of ordered microporous films with water as templates from poly(D,L-lactic-co-glycolic acid) solution," Journal of Applied Polymer Science, vol. 90, no. 7, pp. 1846-1850, 2003.

[15] O. Pitois and B. François, "Formation of ordered microporous membranes," European Physical Journal B, vol. 8, no. 2, pp. 225-231, 1999.

[16] J. Peng, Y. Han, J. Fu, Y. Yang, and B. Li, "Formation of regular hole pattern in polymer films," Macromolecular Chemistry and Physics, vol. 204, no. 1, pp. 125-130, 2003.

[17] B. François, Y. Ederlé, and C. Mathis, "Honeycomb membranes made from C60(PS)6," Synthetic Metals, vol. 103, no. 1-3, pp. 2362-2363, 1999.

[18] P. Jiang, X. Y. Hu, H. Yang, and Q. H. Gong, "Fabrication of two-dimensional organic photonic crystal microcavity," Chinese Physics Letters, vol. 23, no. 7, pp. 1813-1815, 2006.

[19] S. Matsushita, R. Fujiwara, and M. Shimomura, "Calculation of photonic energy bands of self-assembled-type $\mathrm{TiO}_{2}$ photonic crystals as dye-sensitized solar battery," Colloids and Surfaces A, vol. 313-314, pp. 617-620, 2008.

[20] A. Tsuruma, M. Tanaka, N. Fukushima, and M. Shimomura, "Morphological changes in neurons by self-organized patterned films," e-Journal of Surface Science and Nanotechnology, vol. 3, pp. 312-315, 2005.

[21] H. Yabu, M. Takebayashi, M. Tanaka, and M. Shimomura, "Superhydrophobic and lipophobic properties of selforganized honeycomb and pincushion structures," Langmuir, vol. 21, no. 8, pp. 3235-3237, 2005.

[22] M. Tanaka, K. Nishikawa, H. Okubo et al., "Control of hepatocyte adhesion and function on self-organized honeycombpatterned polymer film," Colloids and Surfaces A, vol. 284-285, pp. 464-469, 2006.

[23] H. Sunami, E. Ito, M. Tanaka, S. Yamamoto, and M. Shimomura, "Effect of honeycomb film on protein adsorption, cell 
adhesion and proliferation," Colloids and Surfaces A, vol. 284285, pp. 548-551, 2006.

[24] Y. Fukuhira, H. Kaneko, M. Yamaga, M. Tanaka, S. Yamamoto, and M. Shimomura, "Effect of honeycomb-patterned structure on chondrocyte behavior in vitro," Colloids and Surfaces A, vol. 313-314, pp. 520-525, 2008.

[25] K. Arai, M. Tanaka, S. Yamamoto, and M. Shimomura, "Effect of pore size of honeycomb films on the morphology, adhesion and cytoskeletal organization of cardiac myocytes," Colloids and Surfaces A, vol. 313-314, pp. 530-535, 2008.

[26] Y. Hirai, H. Yabu, and M. Shimomura, "Electroless deposition of zinc oxide on pincushion films prepared by selforganization," Colloids and Surfaces A, vol. 313-314, pp. 312 $315,2008$.

[27] M. Yoshida, "Study of equation of state using laser-induced shock-wave compression 1. Generation and properties of laser-induced shock waves," Journal of Plasma and Fusion Research, vol. 80, no. 6, pp. 427-431, 2004.

[28] M. Born and E. Wolf, Principle of Optics, Pergamon, 1964. 

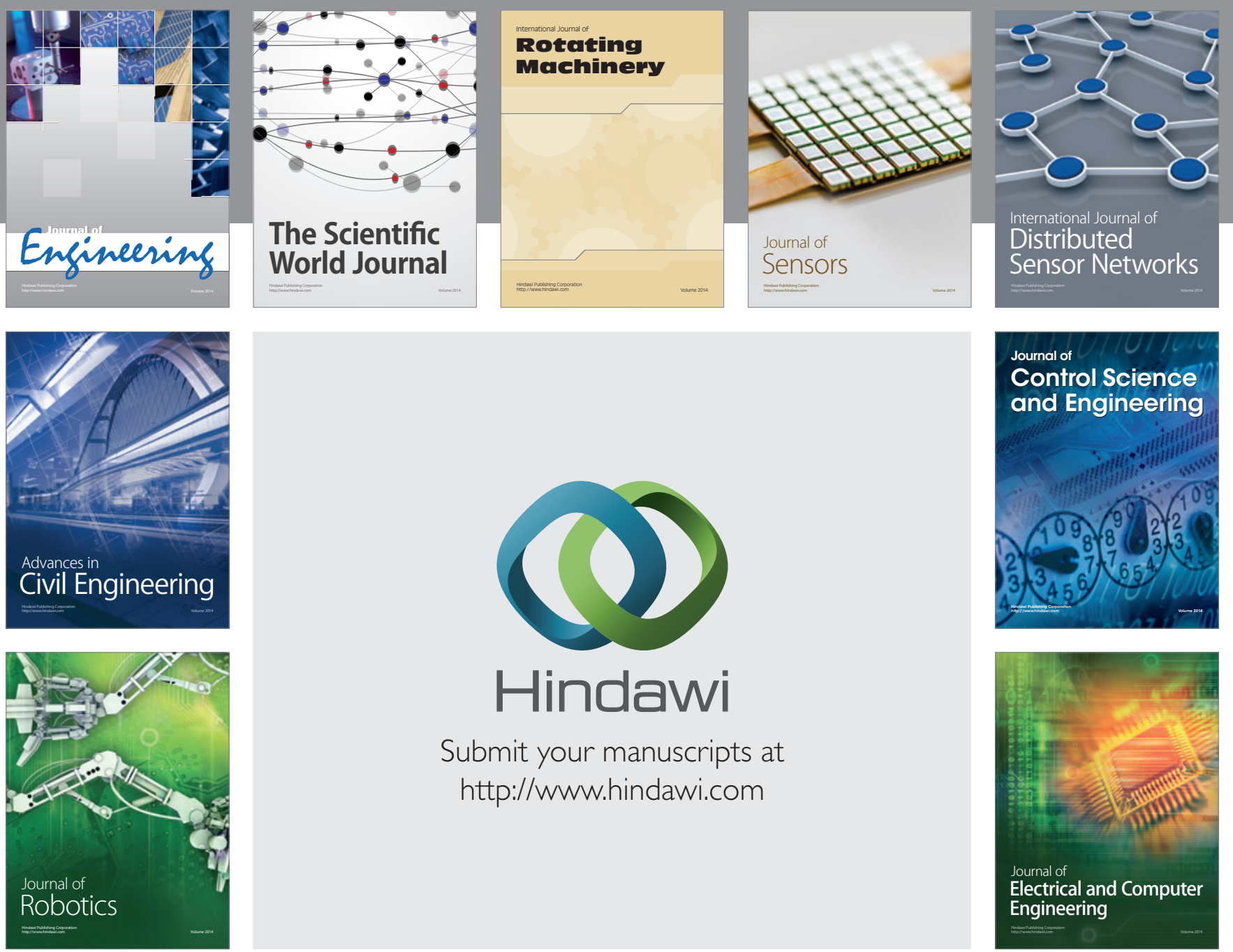

Submit your manuscripts at

http://www.hindawi.com
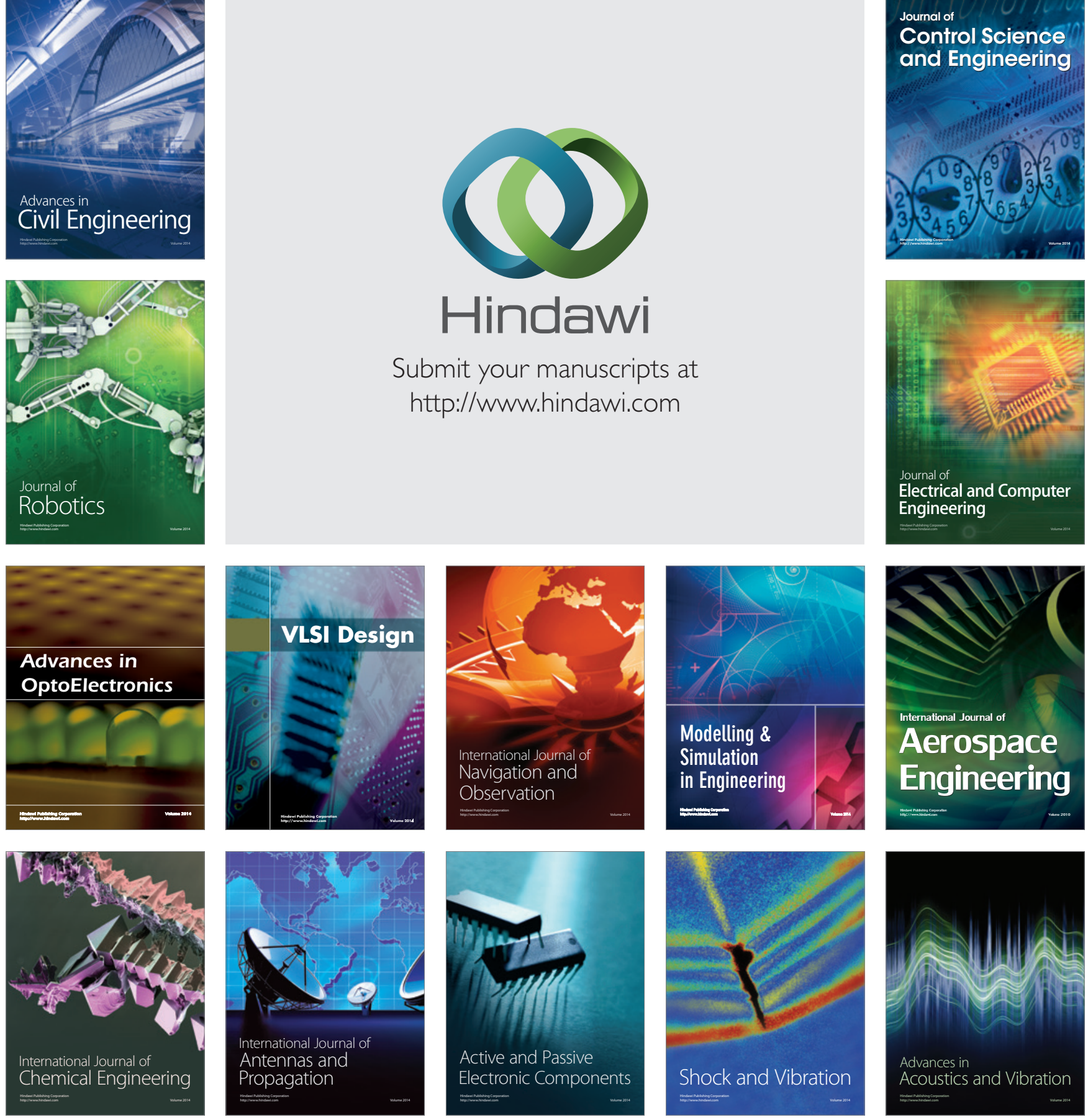